\title{
MARKETING SOCIAL E INFEÇÃO POR VIH/SIDA NAS PESSOAS COM MAIS DE 50 ANOS
}

\author{
Ana Isabel de Almeida Ribeiro* \\ Rui Martins ${ }^{\dagger}$ \\ Paulo Almeida Pereira
}

\begin{abstract}
RESUMO: O Marketing Social tem como finalidade a mudança de comportamentos, o estimular da consciência social em cada indivíduo, obrigando à adoção de novos hábitos, importantes para a pessoa e para a sociedade. O seu crescimento torna-se maior e tão mais importante, quanto maior é a preocupação com os problemas sociais, como é o caso da infeção por VIH/SIDA.

Este é um dos temas que mais preocupa a sociedade atual, não só pelas proporções que tem tomado ao longo do tempo, com o aumento da morbilidade, mas também por todos os custos económicos e socias gerados por ela.

Estudos recentes revelam um aumento exponencial desta síndrome na população com mais de 50 anos, podendo, o marketing social, ser utilizado como estratégia para dirimir este facto.

A investigação em curso teve como objetivo principal discutir e obter informação sobre a relação das duas componentes abordadas, o Marketing Social e a infeção por VIH/SIDA, nos cidadãos comuns com mais de 50 anos, tendo chegado à conclusão que os comportamentos de risco são superiores ao expectável, sendo a educação para a saúde, a consciencialização da doença e a mudança de comportamentos e atitudes as ferramentas mais eficazes para o seu controlo, conseguindo-as facilmente através do marketing social.
\end{abstract}

Palavras-chave: Marketing Social, Infeção por VIH/SIDA em cidadãos com mais de 50 anos.

\footnotetext{
* Enfermeira no Centro Hospitalar Tondela-Viseu (Viseu). Mestre em Gestão Especialização em Gestão e Administração de Unidades de Saúde, pela Universidade Católica Portuguesa, Centro Regional das Beiras. O texto tem por base a sua tese de mestrado. E-mail: anaalmeidaribeiro@ hotmail.com

$\dagger$ Associação Dianova Portugal, Assistente Convidado da Universidade Católica Portuguesa, Centro Regional das Beiras. E-mail: rui.martins@dianova.pt

* Prof. Auxiliar - Departamento de Economia, Gestão e Ciências Sociais - Pólo de Viseu do Centro Regional das Beiras da Universidade Católica Portuguesa. E-mail: ppereira@crb.ucp.pt.
} 


\title{
Ana Isabel de Almeida Ribeiro, Rui Martins e Paulo Almeida \\ Pereira
}

\begin{abstract}
The Social Marketing aims to change behaviors, encourage social awareness in each individual, forcing the adoption of new habits that are important for the person and society. Their growth becomes larger and even more significant, The higher the concern with social problems, such as infection by the HIV / AIDS. This is one of the issues that most concern today's society, not only by the proportions that has taken over time, with increased morbidity, but also by all the economic and social costs generated by it. Recent studies show an exponential increase of this syndrome in the population over 50 years, and social marketing could be used as a strategy to resolve this. The current research aimed to discuss and obtain information on the relationship of the two components addressed, Social Marketing and HIV infection / AIDS, in common with citizens over 50 years, having come to the conclusion that risk behaviors are higher the expected, and the health education, disease awareness and changing behaviors and attitudes are the most effective tools for monitoring, managing them easily through social marketing.
\end{abstract}

Keywords: Social Marketing, HIV Infection / AIDS in Ordinary citizens over 50 years

\section{INTRODUÇÃO}

A saúde pública tem como objetivos a promoção da saúde física e mental através da organização de ações na comunidade no sentido de evitar doenças, permitindo a longevidade dos indivíduos com a melhor qualidade de vida possível.

Tem-se vindo a verificar um aumento das problemáticas sociais, às quais a saúde pública, no sentido lato, não tem conseguido dar resposta. A sua solução requer uma mudança social, a alteração do comportamento e das atitudes da população, a transformação de práticas contraproducentes em proveitosas, que confluam no bem da sociedade.

É neste sentido que surge o marketing social, cuja especificidade está assente no seu objeto, a consciência social, e nos objetivos a que se propõe, despertar consciências e alterar comportamentos e atitudes (Lindon et al, 2008).

A sua aplicação é, no entanto, dificultada pela capacidade de adesão individual à mudança de comportamentos, uma vez que estamos a trabalhar com o ser mais complexo, o Homem.

A infeção por VIH/SIDA é uma problemática atual, transversal a toda a população, com impacto económico, social e político. Está igualmente a aumentar numa faixa etária pouco informada acerca dos perigos de contágio, principalmente devido à falta de campanhas de sensibilização que visem uma atitude defensiva, os cidadãos com mais de 50 anos. 
A constatação deste problema levou-nos à realização de um estudo exploratório que nos permitisse recolher e analisar toda a informação acerca da relação infeção VIH/SIDA em pessoas com mais de 50 anos e marketing social.

\section{MARKETING SOCIAL}

"Num contexto de progressiva competitividade, desregulamentação, liberalização dos fluxos económicos e evolução dramática no comportamento dos consumidores, as empresas que procuram manter um crescimento rápido, mas sustentado numa rendibilidade superior à do mercado têm tomado gradual consciência da importância do marketing como peça fundamental no seu desenvolvimento" (Belmiro de Azevedo, citado por Lindon et al, 2008, p. 432).

Este cenário é transversal a todos os sectores económicos e sociais, desta forma, quando o projetamos, por exemplo, para a saúde, somos orientados para uma área emergente do Marketing: o Marketing Social.

O marketing social foi definido, em 1971, por Kotler e Zaltman como "o desenho, a implementação e o controlo de programas para influenciar a aceitação de ideias sociais, que implica o planeamento do produto, do preço, da comunicação e da distribuição e exige uma prévia pesquisa de mercado" (Kotler e Zaltman, 1971, p.5).

Temas como o tabagismo, controlo de infeção entre profissionais de saúde, prevenção rodoviária e outros, que implicam mudanças de comportamento, têm utilizado o marketing social de forma eficaz e reiterada (Maha et al., 2006), sendo este maioritariamente discutido, não só através da promoção da saúde, como também da garantia de acesso à mesma.

No marketing social pretende-se a "sensibilização e educação da população para questões essenciais como a prevenção de doenças, o acompanhamento periódico e preventivo do estado de saúde, sintomas de alerta e opções de tratamento" (Rui Ribeiro, 2009, Abril/Junho, p.9).

Desta forma, ambiciona-se assim, estimular de forma decisiva e conscienciosa, os comportamentos individuais a fim de se obter um benefício para a sociedade, residindo o seu principal obstáculo, na capacidade de estimulação da consciência social de cada um, para que este, para além da adesão à causa, mude, efetivamente, o seu comportamento.

\subsection{Marketing Social de Saúde}

A saúde é influenciada por factores de ordem económica, social, política e, portanto, culturais. Assim, é mandatório a adoção de medidas 
de saúde pública, sejam elas a preocupação com a educação, a higiene pessoal, o saneamento básico, o controlo das doenças infeciosas, ou os mecanismos de proteção social, entre outros. Estes esforços, se equilibrados, têm um impacto preferencial na promoção da qualidade de vida, na prevenção das doenças e na melhoria da esperança de vida, o mesmo será dizer ganhos globais em matéria de saúde (Nunes e Rego, 2002).

Falar de Marketing na Saúde, ainda hoje, não é bem aceite pela maioria dos cidadãos comuns, isto porque existe a ideia errada de que com o marketing se pretende vender algo.

No entanto, este deve ser um aliado e não um inimigo, deve ser utilizado estrategicamente pelos gestores das diferentes unidades de saúde, pois o marketing social, como referido anteriormente, tem como finalidade a mudança de comportamentos individuais, através de um conjunto de ações bem dirigidas.

A crise que vivemos leva-nos a refletir sobre o atual sistema de saúde em portugal, cuja índole gratuita é de extrema importância, mas que também leva a uma desresponsabilização do utente pela própria saúde, não só porque, culturalmente, a nossa população tende a classificar como mau tudo o que é gratuito, mas também porque os profissionais de saúde propendem a infantilizar o utente, querendo, pela via mais fácil, obrigar à adoção de determinadas atitudes e ou medidas, relegando para segundo plano a modificação de comportamentos, quando este deve estar na génese da prestação dos cuidados.

O Marketing é uma ferramenta essencial para o desenvolvimento de programas de Saúde pública, contribuindo, não só para a alteração de comportamentos da população, profissionais de saúde, e sua corresponsabilização, mas também para uma melhor qualidade dos serviços de saúde (Goldstein e Libb, 2009).

\section{INFEÇÃO POR VIH/SIDA NA POPULAÇÃO COM MAIS DE 50 ANOS}

A infeção por VIH/SIDA é uma problemática global, transversal a todos os estratos sócio económicos e faixas etárias, podendo ser definida como "uma doença infeciosa crónica, causada por um de dois vírus (VIH-1/VIH-2 (Vírus da Imunodeficiência Humana 1 e 2)), caracterizada principalmente pela diminuição progressiva da imunidade dos infetados" (Barroso, A., 2008), cuja evolução de vários anos é dividida em três fases principais: a infeção primária ou primo 
infeção, o período de latência e a fase de SIDA (Síndrome da Imunodeficiência Adquirida)

A primo infeção pode ser sintomática ou assintomática, é de curta duração e ocorre pouco após o contágio, passando para uma fase assintomática, que consiste num período muito mais longo. Segue-se o período de latência, cuja sintomatologia é comum a outras patologias (cefaleias, sensação de mal-estar), evoluindo durante cerca de 8 a 10 anos. Na última fase há o surgimento de doenças, sendo estas cada vez mais graves, devido à falha de imunidade, surgindo as infeções oportunistas (Barroso, A., 2008).

A SIDA não é, portanto, uma doença mas uma síndrome, ou seja, um conjunto de sintomas e sinais que não dizem respeito apenas a uma doença. A transmissão do vírus da imunodeficiência humana efetua-se por três vias principais: sexual; sanguínea e materno-fetal.

Com enorme impacto social e político, não só devido aos medos do público quanto à infeção por VIH, mas também pelos crescentes custos económicos, a seropositividade para o VIH, bem como a sua evolução no sentido de SIDA, que tem vindo a distanciar-se da metáfora da "espada de Damócles", que a conota com uma sentença de morte a curto prazo, mas antes e devido em grande parte à evolução das terapias medicamentosas, como doença crónica (Erlen et al., 2001).

Atualmente o tratamento anti retrovírico diminui a taxa de mortalidade e aumenta a esperança média de vida dos portadores do vírus, sendo assim, possível viver com VIH durante largos anos e com relativa qualidade de vida.

A evolução mais lenta da doença, não lhe retirou a gravidade enquanto problema de saúde pública, no entanto a perda do seu estigma como "sentença de morte", refletiu-se na perda da visibilidade da infeção nos media e na consciência das pessoas. A comunicação passou a focar, essencialmente, a facilidade de transmissão do vírus e os comportamentos que garantem a sua prevenção. Assim, passaram a existir, fundamentalmente, dois tipos de mensagem, uma que proclama a prática de sexo seguro e alerta para os comportamentos de risco; a outra que combate o estigma e a discriminação relativa aos portadores da síndrome (Oliveira, 2007).

Estudos recentes revelam um crescimento exponencial de novos casos de infeção por VIH/SIDA na população com mais de 50 anos, nos países desenvolvidos, incluindo Portugal (Casau, 2005, Dias, A., 2005, Xará, S. et al, 2006), surgindo um tipo de doente até então inexistente, o indivíduo em processo de envelhecimento com patologia associada, seropositivo e a ter de lidar com a toxicidade medicamentosa da terapia antirretroviral combinada (Dias, A., 2005). 


\section{Ana Isabel de Almeida Ribeiro, Rui Martins e Paulo Almeida \\ Pereira}

A infeção VIHISIDA nas pessoas com mais de 50 anos não é frequentemente detetada de forma precoce. (Knoel et al, 2002). Isto devese essencialmente a dois factos, um de índole social gerado pela falsa crença dos clínicos, de que nos idosos não existe SIDA e de que este grupo etário não apresenta uma vida sexual ativa. Aliás, estudos recentes demonstram precisamente o contrário, a via sexual é a principal forma de contágio na faixa etária em análise. (Cloud et al, 2003).

$\mathrm{O}$ aparecimento de novos fármacos que promovem uma vida sexual ativa contribui largamente para este facto. Um outro fator preponderante na infeção VIH $\backslash S I D A$, relaciona-se com outras doenças crónicas próprias do processo de envelhecimento que mascaram os sintomas de SIDA e que induzem um diagnóstico errado ou de certo modo inconclusivo. (Stoff et al, 2004).

Convém também lembrar que esta é uma faixa etária ainda não muito informada acerca dos perigos de contágio devido à ausência de campanhas de sensibilização que visem uma atitude defensiva. Entre outras, esta é porventura a melhor explicação para a fraca adesão ao preservativo. Outros aspetos como os valores religiosos, ou ainda, motivados pelo facto da parceira ter já atingido a menopausa, não devem ser ignorados. (Stoff et al, 2004).

Segundo Dolder et al (2004), outra causa, diz respeito à existência de violência doméstica na vida conjugal. Esta realidade propicia encontros sexuais anónimos e o recurso à prostituição, o mesmo sucedendo em situação de viuvez, sobretudo nos homens.

O diagnóstico de infeção pelo VIH/SIDA em idosos é feito geralmente quando referem sintomatologia ou quando são hospitalizados, encontrando-se normalmente já num estádio avançado da doença. Um diagnóstico precoce é fundamental pois apesar de se encontrarem num estádio avançado, o uso da terapia anti retrovírica nestes indivíduos contribui para a diminuição da morbilidade e mortalidade.

Nos indivíduos com idade acima dos 50 anos é urgente a utilização de medidas preventivas e de educação para a saúde eficaz, uma vez que só um sexto dos indivíduos com comportamentos de risco utiliza proteção (Chaves, 2009).

A Educação para a saúde nas pessoas com mais de 50 anos deve ter em consideração a sua história de vida, a sua relação com os outros, auxiliando-o a ter tomadas de decisão conscientes e livres que o conduzam a comportamentos promotores de saúde. Esta não se deve pois, a partir desta idade a vulnerabilidade a problemas de saúde e sociais aumenta, devendo-se privilegiar as atividades que tendem a melhorar as 
suas capacidades e autonomia e facilitar o acesso aos recursos disponíveis ao mesmo tempo que se responsabiliza pela sua própria saúde.

\subsection{Marketing Social na prevenção da infeção por VIH/SIDA}

A prevenção da infeção pelo VIH/SIDA apenas pode ser conseguida através da redução dos meios de propagação do vírus. A prevenção aparece, assim, como a única arma atualmente disponível nesta batalha (Tubiana R., 2005).

A educação para a saúde justifica-se pela urgência assente em vários pressupostos, tais como informar, aconselhar, orientar e sobretudo educar a população, em particular o grupo etário em análise, para a saúde e para uma sexualidade feliz e responsável, cabendo aos profissionais de saúde torná-la objeto de preocupação e de decisão consciente de todos.

Para dar resposta a estes problemas emerge do Marketing, o Marketing Social! "O importante no marketing social é a capacidade dos seus agentes de apelarem à consciência social, cívica e solidária dos cidadãos. Por isso, as campanhas sociais são concebidas para educar e para prevenir" (Lindon et al., 2008, p.589).

Sendo uma doença ligada aos comportamentos, torna-se emergente a necessidade de um sistema de saúde integrado, centrado na promoção e prevenção da saúde, consubstanciando-se o Marketing Social como a estratégia mais adequada neste sentido.

\section{MATERIAL E MÉTODOS}

Realizámos um estudo exploratório, que tem como objetivo basilar discutir e obter informação sobre a relação das duas componentes abordadas, o Marketing Social e a infeção por VIH/SIDA, nos cidadãos comuns com mais de 50 anos.

A estratégia metodológica aplicada foi a quantitativa, tendo sido realizado um questionário anónimo.

A amostra é constituída por 300 inquiridos, portugueses, com mais de 50 anos, na sua maioria (49\%) entre os 61 e 70 anos, 57\% do sexo masculino e $43 \%$ do sexo feminino, $60 \%$ da amostra pertence ao meio urbano e os restantes $40 \%$ ao meio rural, sendo $80 \%$ casados. Quanto à escolaridade na sua pluralidade $(43,7 \%)$ frequentaram o $1^{\circ}$ ciclo (até ao $4^{\circ}$ ano), encontrando-se os restantes distribuídos pela frequência no Ensino Superior, Secundário, $3^{\circ}$ ciclo, $2^{\circ}$ ciclo e uma minoria $(1,7 \%)$ que não sabe ler nem escrever. $64 \%$ dos inquiridos encontra-se já reformado. 


\section{RESULTADOS}

Os resultados dos 300 inquéritos realizados sugerem que, apesar do conhecimento existente sobre a infeção por VIH/SIDA, os comportamentos de risco adotados são superiores ao expectável, traduzidos pelo Gráfico 1, que evidencia as respostas dadas às perguntas (análise descritiva): O vírus VIH é o causador da SIDA (1); O vírus da SIDA pode ser transmitido por sabonetes, toalhas e assentos de sanita (2); $O$ vírus da SIDA pode ser transmitido por relações sexuais, sangue e de mãe para filho durante a gravidez ou parto (3); O uso de preservativo nas relações sexuais pode impedir a transmissão do vírus da SIDA (4); O uso da mesma seringa e agulha por diversas pessoas pode transmitir SIDA (5); A SIDA é uma doença que ocorre somente em homossexuais, prostitutas e toxicodependentes (6); Os indivíduos com idade acima dos cinquenta anos não se devem preocupar com a SIDA pois ela só atinge os mais jovens (7); Utiliza sempre preservativo em relações sexuais extraconjugais (se aplicável/ se as tem)? (8); Se soubesse que tinha contraído VIH/SIDA, comunicaria o facto ao seu conjugue? (9); Já ouviu falar em Marketing Social? (10); O Marketing Social pretende despertar a consciência social, modificar atitudes e alterar comportamentos? (11); As campanhas que passam nos meios de divulgação (televisão, posters, cartazes, panfletos...) sobre SIDA ajudam a prevenir a doença? (12); Já assistiu a alguma campanha/ação, que visasse a mudança de comportamentos, relativamente à SIDA, nas pessoas com mais de 50 anos? (13).

\section{Gráfico I}

Análise Descritiva

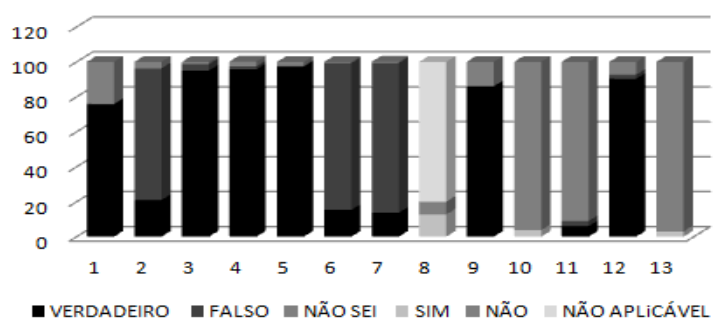

O cruzamento das diferentes variáveis permitiu-nos inferir que:

- tanto os inquiridos do sexo feminino, como os inquiridos do sexo masculino, demonstram um bom conhecimento sobre a infeção por VIH/SIDA, não havendo relação entre as duas variáveis; 
- os inquiridos do sexo masculino apresentam mais comportamentos de risco face à infeção por VIH/SIDA, que os inquiridos do sexo feminino, principalmente no que respeita ao uso de preservativo nas relações sexuais extraconjugais;

- são os inquiridos do meio urbano que aglomeram a maior percentagem de respostas corretas, demonstrando, assim, mais conhecimento sobre a infeção por VIH/SIDA, comparativamente aos inquiridos do meio rural;

- os inquiridos do meio rural apresentam mais comportamentos de risco face à infeção por VIH/SIDA, do que os inquiridos do meio urbano;

- são os inquiridos divorciados ou separados os que apresentam mais comportamentos de risco face à infeção por VIH/SIDA, no que respeita ao uso de preservativo nas relações sexuais extraconjugais. No entanto, a maioria dos inquiridos, independentemente do estado civil, se contraísse VIH/SIDA, comunicaria o facto ao seu cônjuge;

- os inquiridos que ostentam um conhecimento sobre a infeção por VIH/SIDA mais fidedigno, são os que apresentam menos comportamentos de risco face à mesma;

- a maioria da nossa amostra, independentemente do conhecimento que tenha, ou não, da infeção por VIH/SIDA, não demonstra conhecimento sobre o Marketing Social. Contudo, os inquiridos que apresentam conhecimento da infeção por VIH/SIDA, são os que consideram que as campanhas que passam nos meios de divulgação (televisão, posters, cartazes, panfletos...) sobre SIDA, ajudam a prevenir a doença, abrindo assim as vias de comunicação no que respeita ao Marketing Social;

- paralelamente ao que sucede no ponto anterior, a maioria dos inquiridos, quer apresente comportamentos de risco face à infeção por VIH/SIDA, ou não, não demonstra conhecimento sobre o Marketing Social. Todavia, são os inquiridos que evidenciam ausência de comportamentos de risco, que consideram que as campanhas que passam nos meios de divulgação (televisão, posters, cartazes, panfletos...) sobre SIDA, ajudam a prevenir a doença.

\section{DISCUSSÃO E CONCLUSÕES}

Quando olhamos para os resultados obtidos não podemos deixar de pensar em toda a pesquisa bibliográfica, que é reforçada por eles.

Dolder et al. (2004), afirmam a propensão para encontros sexuais anónimos e recurso à prostituição nos homens com mais de 50 anos, 


\section{Ana Isabel de Almeida Ribeiro, Rui Martins e Paulo Almeida \\ Pereira}

sendo estes que, efetivamente, adotam mais comportamentos de risco face à infeção por VIH/SIDA.

A utilização de medidas preventivas e educacionais no que respeita á infeção por VIH/SIDA, nos indivíduos com idade acima dos 50 anos é primordial, constituindo a singular arma disponível nesta batalha (Chaves, 2009 e Tubiana, 2006). Facto este secundado pela nossa amostra, uma vez que, independentemente do seu conhecimento sobre o Marketing Social, considera que as campanhas que passam nos meios de divulgação (televisão, posters, cartazes, panfletos...) sobre SIDA, ajudam a prevenir a doença.

Desta forma, e apesar da maioria da amostra em estudo não conhecer o Marketing Social, é consentânea a considerar que as campanhas que passam nos meios de divulgação (televisão, posters, cartazes, panfletos...) sobre SIDA, ajudam a prevenir a doença, o que vem de acordo com a máxima e objetivo final do mesmo.

Aferimos, também, que são os inquiridos do meio urbano que manifestam superior conhecimento sobre a infeção por VIH/SIDA, enquanto os do meio rural demonstram a existência de mais comportamentos de risco, face à mesma infeção. Consideramos que o facto se deve a um maior nível de informação existente nas cidades, não só pelas campanhas existentes em outdoors, mas também por causa do trabalho efetuado por profissionais de saúde e educativos nas escolas, centros de saúde, centros sociais, etc., patenteando a importância da informação e do Marketing Social.

Segundo Stoff et al (2004), a ausência de campanhas de sensibilização direcionadas para esta faixa etária, torna parca a sua informação sobre os perigos de contágio.

Contudo, o estudo realizado, ao contrário da revisão bibliográfica, reporta-nos para um efetivo conhecimento sobre a infeção por VIH/SIDA, na maioria da nossa amostra. Este facto contribui para uma diminuição dos comportamentos de risco adotados face à mesma infeção, sendo estes, ainda assim, mais presentes do que o espectável. O que nos leva a aferir a necessidade, secundada por Stoff et al. (2004) e Chaves (2009) de campanhas de sensibilização.

Consideramos, então, que a única forma eficaz de proteção contra a doença, continua a ser a consciencialização sobre o risco de infeção, modificação de atitudes e alteração de comportamentos, podendo proceder-se à divulgação dos mesmos, de uma forma mais eficaz, através do Marketing Social, que cumpria, assim, os seus objetivos.

Torna-se assim emergente para, políticos, profissionais de saúde, administradores de entidades de saúde, entre outros, repensarem a sua 
conduta atual e apostarem em campanhas de prevenção levando essas mesmas campanhas à população e à comunidade de forma pró-ativa.

\section{FONTES E BIBLIOGRAFIA}

Casau N. C.(2005). Perspective on HIV infection and aging: Emerging Research on the horizon. AIDS. Vol. 41. $\mathrm{N}^{\mathrm{o}} 6$, pp 855-863.

Cloud, G., Browne, R., Salooje, N., McLean, K. (2003). Newly diagnosed $H I V$ infection in an octogerian: the elderly are not "immune". Age and ageing. Vol. 32. No 3, pp 353-354.

Dolder, C., Pattersona, T., Jeste, D. (2004). HIV, psychosis and aging: past, present and future. AIDS. Vol. 18, pp 35-42.

Knodel J,et al (2002) The impact of the AIDS epidemic in older persons. AIDS. Vol. 16. No 4, pp. 77-83.

Kotler, P. e Zaltman, G. (Julho, 1971). Social Marketing: an approach to planned social change. Journal of Marketing. Vol. 35, pp. 3-12.

Kotler, Philip e Roberto, Eduardo L. (1992). Marketing Social. Estratégias para alterar o comportamento Público. Editora campus. Rio de Janeiro.

Kuazaqui, E. e Tanaka, L. (2008). Marketing e Gestão Estratégica de Serviços de Saúde. Thomson Learning. São Paulo.

Lindon, D, Lendrevie, J., Levy, J., Dionísio, P., Rodrigues, J. (2008). Mercator XXI: Teoria e prática do Marketing. Publicações Dom Quixote. Lisboa.

Mah, M., Deshpande, S. e Rothschild, M. (Setembro, 2006). Social Marketing: a behavior change technology for infection control. American Journal of Infection Control. Vol. 34. No 7, pp. 452-457.

Moreira, Paulo K (2007). Políticas de Saúde. Ensaios para um Debate Nacional. Edições Universidade Fernando Pessoa. Porto.

Nunes, Rui e Rego, Guilhermina (2002). Prioridades na saúde. MacGraw-Hill. Lisboa.

Oliveira, A. (2007). O Uso e Não Uso de Preservativo numa População Jovem. Contributo para a compreensão dos factores que condicionam a adesão aos mecanismos de prevenção do VIH / SIDA. Dissertação de Mestrado. Coimbra: Faculdade de medicina da Universidade de Coimbra.

Ribeiro, R. (2009, Abril/Junho). Entrevista com .... Dianova EXIT, pp. 810.

Stoff, D., Khalse, J., Monjan, A., Portegies, P. (Janeiro, 2004). Introduction: HIV/AIDS and Aging. AIDS. Vol. 18, pp. 1-2.

Tubiana R. (Novembro, 2005). HIV management in Africa. Presse Med. Vol. 19. N³4, pp. 1597-1604. 


\section{Internet:}

Barroso, Ana (2008). Conhecimento dos Enfermeiros face à Infecção VIH/SIDA. http://hdl.handle.net/10316/10774, consultado a 16.02.2010.

Chaves, C. (2009). Sida e o Idoso. Metodologias educativas de âmbito preventivo. $\quad \mathrm{http}: / / \mathrm{www} . \mathrm{ipv} . \mathrm{pt} / \mathrm{millenium} / \mathrm{millenium32/9.pdf}$, consultado a 03.03.2010.

Dias, A., Fonseca, S., Renca, P., Silva, E. (2005). A Infecção VIH/SIDA na População com mais de 50 anos. Análise estatística da População com mais de 50 anos infetada em Portugal.

http://www.aidscongress.net/pdf/50_anos_abstract_233_comunic_266.pd $\mathrm{f}$, consultada a 02.02.2010.

Erlen, J., Sereika, S., Cook, R., Hunt, S. (2001). Adherence to Antiretroviral Therapy Among Women With HIV Infection.

http://www3.interscience.wiley.com/journal/118920729/abstract?CRETR $\mathrm{Y}=1 \&$ SRETRY $=0$, consultado a 02.01.2010.

Goldstein, Nacy e Libb, Alli (2009). Good Marketing: Marketing for Social Good. Journal of Marketing. http://www.marketingpower.com/ResourceLibrary/Pages/Webcasts/ Marketing_for_Social_Good_092909.aspx?sq=social+marketing, consultado a 12.05.2010.

Xará, S., Dias, I., Mota, M. (2006). Nutrição e VIH: Particularidades no Idoso. http://www.aidscongress.net/pdf/298.pdf, consultado a 03.03.2010. 